\title{
Attachment and growth of anchorage-dependent cells on a novel, charged-surface microcarrier under serum-free conditions
}

\author{
James Varani*, Felicia Piel, Sean Josephs, Ted F. Beals \& William J. Hillegas \\ ${ }^{1}$ Department of Pathology, The University of Michigan, 1301 Catherine Road, P.O. Box 0602, Ann Arbor, \\ MI 48109 U.S.A. \\ E-mail:varani@umich.edu \\ ${ }^{2}$ SoloHill Engineering, Inc., Ann Arbor, MI, U.S.A.
}

Received 25 August 1998; accepted 25 August 1998

Key words: cell lines, diploid fibroblasts, microcarriers, serum-free culture

\begin{abstract}
The present study describes a novel microcarrier substrate consisting of a swellable, copolymer of styrene and divinylbenzene, derivatized with trimethylamine. The co-polymer trimethylamine microcarriers support the growth of a number of different cell lines - Madin Darby Bovine Kidney, Madin-Darby Canine Kidney, Vero and Cos-7 - under serum-free conditions, and human diploid fibroblasts in serum-containing medium. Cells attach to the co-polymer trimethylamine microcarriers as rapidly as they attach to other charged-surface microcarriers (faster than they attach to collagen-coated polystyrene microcarriers) and spread rapidly after attachment. All of the cells examined grow to high density on the co-polymer trimethylamine microcarriers. Furthermore, cells are readily released from the surface after exposure to a solution of trypsin/EDTA. In this respect, the co-polymer trimethylamine microcarriers are different from other charged-surface microcarriers. Madin-Darby Bovine Kidney cells grown on this substrate support production of vaccine strain infectious bovine rhinotracheitis virus as readily as on other charged-surface or collagen-coated microcarriers. Thus, the co-polymer trimethylamine microcarriers combine the positive characteristics of the currently available charged-surface and adhesion-peptide coated microcarriers in a single product. The viral vaccine production industry is undergoing considerable change as manufacturers move toward complete, animal product-free culture systems. This novel substrate should find application in the industry, especially in processes which depend on viable cell recovery.
\end{abstract}

Abbreviations: MDBK - Madin Darby Bovine Kidney Cells; MDCK - Madin Darby Canine Kidney Cells; CPTMA - Co-polymer trimethylamine; DMEM-FBS - Dulbecco's Modified Medium Essential Medium of Eagle with $10 \%$ fetal bovine serum; IBR - Infectious bovine rhinotracheitis virus.

\section{Introduction}

The ideal microcarrier should i) support rapid cell attachment and spreading; ii) support high-density cell growth; iii) not interfere with the elaboration or secretion of metabolic products; and iv) allow cells to be easily-detached. These characteristics should be expressed under serum-free conditions as well as in the presence of low serum-containing medium. Any product that combines these features along with low

\footnotetext{
* Author for all correspondence.
}

manufacturing costs, long shelf-life, and ease of handling will find acceptance in the biologicals industry for the large-scale cultivation of anchorage-dependent cells.

There are two basic strategies for achieving the ideal microcarrier. One involves covalently linking a strongly charged surface moiety such as diethyl amino ethyl (DEAE) to a porous or semi-porous neutral substrate such as dextran or polysytrene plastic (Van Wezel, 1967). The other involves coating the substrate surface with an adhesion-supporting extracellu- 
lar matrix component such as collagen or fibronectin - or with synthetic peptides from the attachmentsupporting domains of these matrix components $(\mathrm{Ru}-$ oslahti and Pierschbacher, 1986; Varani et al., 1989; Varani et al., 1993). Cellular attachment to charged surfaces involves electostatic interactions (Ginsburg, 1987) while interactions with adhesion peptides occurs through specific cell surface receptors (Swartz, 1993). In general, charged surfaces support better cell attachment under the high shear-stress conditions present in bioreactor cultures, but once cells have attached, they often spread and begin proliferating more slowly than on collagen-coated microcarriers (Varani et al., 1989; Varani et al., 1995). Furthermore, it is often difficult to harvest viable cells from charged surfaces, while cells are readily released (and, therefore, retain high viability) from matrix-coated materials (Varani et al., 1986). In the present report we describe a novel substrate consisting of a swellable copolymer of styrene and divinylbenzene, derivatized with trimethylamine (CP-TMA). The CP-TMA microcarriers combine the desirable properties of both types of currently-available microcarriers in a single product. This novel substrate should find application in the viral vaccine-manufacturing industry (especially in processes which depend on viable cell recovery) as the industry moves toward complete animal product-free culture systems.

\section{Materials and methods}

\section{Microcarriers}

CP-TMA microcarriers and collagen-coated polystyrene plastic microcarriers were produced by Solohill Labs (Ann Arbor, MI). DEAE-dextran microcarriers (Cytodex I) were obtained from Amersham - Pharmacia Biotechnology (London, UK). All three microcarriers products were prepared for use as indicated by their respective manufacturers. Briefly, the dried collagen-coated polystyrene plastic microcarriers were resuspended in PBS and autoclaved. The CP-TMA microcarriers and DEAE-dextran microcarriers were swelled in PBS and washed several times. Following this, these substrates were sterilized by autoclaving. After sterilization and cooling, all three preparations were rinsed in PBS and brought to a final bead concentration of $50 \mathrm{mg} \mathrm{mL}^{-1}$.

Experiments were conducted in 2-mL plastic culture dishes and in 100-mL, bulb-stirred suspension culture flasks. When plastic culture dishes were used, the surface was first coated with a polyhydroxymethacrylate solution to prevent cell-sticking to the polystyrene plastic. CP-TMA microcarriers were added to the cultures at $25 \mathrm{mg} \mathrm{mL}^{-1}$, providing a surface area of approximately $18 \mathrm{~cm}^{2} \mathrm{~mL}^{-1}$. DEAEdextran and collagen-coated polystyrene microcarriers were used at comparable amounts of surface area.

\section{Cells}

Four different cell lines were used in these studies. These were Madin-Darby Bovine Kidney (MDBK), Vero (African Green Monkey Kidney), Madin-Darby Canine Kidney (MDCK) and Cos-7. All were obtained from the American Type Culture Collection. In addition to the cell lines, low-passage human diploid fibroblasts obtained from neonatal foreskin as described previously (Tamm et al., 1984) were also used. The cells were maintained in monolayer culture using Dulbecco's Modified Minimal Essential Medium of Eagle with Earles Salts, nonessential amino acids and $10 \%$ fetal bovine serum (DMEM-FBS) as culture medium. Incubation was at $37{ }^{\circ} \mathrm{C}$ in an atmosphere containing $95 \%$ air and $5 \% \mathrm{CO}_{2}$. Cell lines were subcultured as required, while human neonatal fibroblasts were isolated from fresh tissue weekly.

\section{Cell attachment and spreading on CP-TMA microcarriers}

Attachment assays were carried out in $2-\mathrm{mL}$ culture dishes. Cells $\left(2.5 \times 10^{5}\right.$ per $\left.\mathrm{mL}\right)$ were added to cultures containing $25 \mathrm{mg}$ per $\mathrm{mL}$ of CP-TMA microcarriers (or an equivalent amount of DEAE-dextran or collagen-coated polystyrene microcarriers) and incubated at $37{ }^{\circ} \mathrm{C}$. After $0.5,1,2$ and $4 \mathrm{hr}$, microcarriers with cells attached were removed. Cells were separated from the microcarriers with trypsin/EDTA and counted. From these counts, the percentage of cells that were attached at each time point was determined. Additionally, microcarriers with attached cells were examined microscopically. Cells that were fully rounded were scored as non-spread, while fully- or partially-flattened cells were scored as spread. The percentage of spread cells was determined from this.

\section{Cell growth}

Growth studies were conducted in 100-mL suspension cultures. Cells $\left(4-5 \times 10^{5}\right.$ per $\left.\mathrm{mL}\right)$ were added to cultures containing $25 \mathrm{mg}$ per $\mathrm{mL}$ of CP-TMA 
at time-zero and incubated. Fifty percent of the culture medium (serum-free Aim V culture medium; [GIBCO, Grand Island, NY] unless otherwise indicated) was replaced every other day. At days 2, 4 and 6 , microcarriers with cells attached were removed. Cells were separated from the microcarriers with trypsin/EDTA and counted to give a total cell count. A fraction of the harvested cells was washed two times, resuspended in DMEM-FBS and plated in plastic cell culture dishes. Four hours later, the cells that had reattached were harvested and counted. Cells that had successfully reattached were considered viable, and from this the percentage of viable cells was determined.

\section{Scanning electron microscopy}

Cells and microcarriers were fixed in situ with $4 \%$ Sorenson's buffered glutaraldehyde and processed for scanning electron microscopy exactly as described previously (Varani et al., 1983). Briefly, this involved post-fixation with $1 \%$ osmium tetroxide buffered scollidine followed by staining with uranyl acetate, subsequent dehydration in graded ethanol - liquid carbon dioxide and finally gold sputter-coated. Specimens were examined using an ISI Super IIIA scanning electron microscope.

\section{Cell detachment assay}

Cells were grown for two days on CP-TMA microcarriers or on the control substrates in serum-free Aim V medium. At the end of the incubation period, the cells were exposed to a solution containing $0.5 \%$ trypsin $(\mathrm{w} / \mathrm{v})$ and $0.5 \mathrm{mM}$ EDTA in $\mathrm{Ca}^{2+}-$ and $\mathrm{Mg}^{2+}$ - free PBS. The cells were incubated in this solution for 5, 10 or $15 \mathrm{~min}$. At each time point, the cells and microcarriers were gently triturated to facilitate cell release. The released cells were counted and a percentage of released cells at each time point calculated.

\section{Production of infectious bovine rhinotracheitis (IBR) virus}

IBR virus (ATCC VR-864) was obtained from the American Type Culture Collection. Virus stocks were prepared in MDBK cells, titered and frozen in liquid nitrogen. To assess virus growth, MDBK cells were grown for one day in 100-mL suspension cultures containing either CP-TMA microcarriers or one of the two control types of microcarriers. Virus was seeded at 0.5 infectious units per cell on day zero. After 1, 2 and 3
Table 1. Attachment and spreading of four different cell lines and human diploid fiboblasts on CP-TMA microcarriers

\begin{tabular}{lll}
\hline Cell type & $\begin{array}{l}\text { Percent attached } \\
\text { (mean } \pm \text { s.d.) }\end{array}$ & $\begin{array}{l}\text { Percent spread } \\
\text { (mean } \pm \text { s.d.) }\end{array}$ \\
\hline MDCK & $89 \pm 10$ & $71 \pm 5$ \\
MDBK & $75 \pm 6$ & $92 \pm 7$ \\
Vero & $94 \pm 8$ & $89 \pm 16$ \\
Cos-7 & $90 \pm 2$ & $55 \pm 6$ \\
Diploid fibroblasts & $70 \pm 5$ & $60 \pm 6$ \\
\hline
\end{tabular}

Cells were added to the microcarriers at time-zero and allowed to attach for a period of one hour. At the end of the incubation period, the percent attached was assessed by direct cell counts made following removal of the cells from the substrate with trypsin/EDTA. The percent spread was determined by assessing cells on individual beads under phase-contrast microscopy and determining the percentage of cells that were flattened out on the bead (spread) and the percentage that were still round and refractile to light (not spread). MDBK = Madin-Darby Bovine Kidney; MDCK = Madin-Darby Canine Kidney; Vero = African Green Monkey Kidney; Diploid fibroblasts are derived from human neonatal foreskin.

days, samples of culture fluids were obtained. The culture fluids were clarified by low-speed centrifugation and the virus quantified in MDBK cell monolayers.

\section{Results}

Attachment of cells to CP-TMA microcarriers: Comparison with attachment to collagen-coated polystyrene microcarriers and to DEAE-dextran microcarriers

In the first series of experiments, MDBK cells were examined for attachment to the experimental microcarriers under serum-free conditions. Attachment to collagen-coated polystyrene microcarriers and DEAEdextran microcarriers was assessed in parallel as controls. As expected, the cells attached rapidly to the DEAE-dextran microcarriers and more slowly to the collagen-coated polystyrene microcarriers (Figure 1). When the CP-TMA microcarriers were examined, MDBK cells attached as rapidly to this substrate as to the DEAE-dextran microcarriers (Figure 1). By one hour, $75-90 \%$ of the cells were attached to both the CP-TMA and DEAE-dextran microcarriers as compared to approximately $50 \%$ to the collagen-coated polystyrene microcarriers (Figure 1). Table 1 summarizes attachment and spreading data for the four different cell lines and the low-passage human diploid fibroblasts on CP-TMA microcarriers. All of the cells 


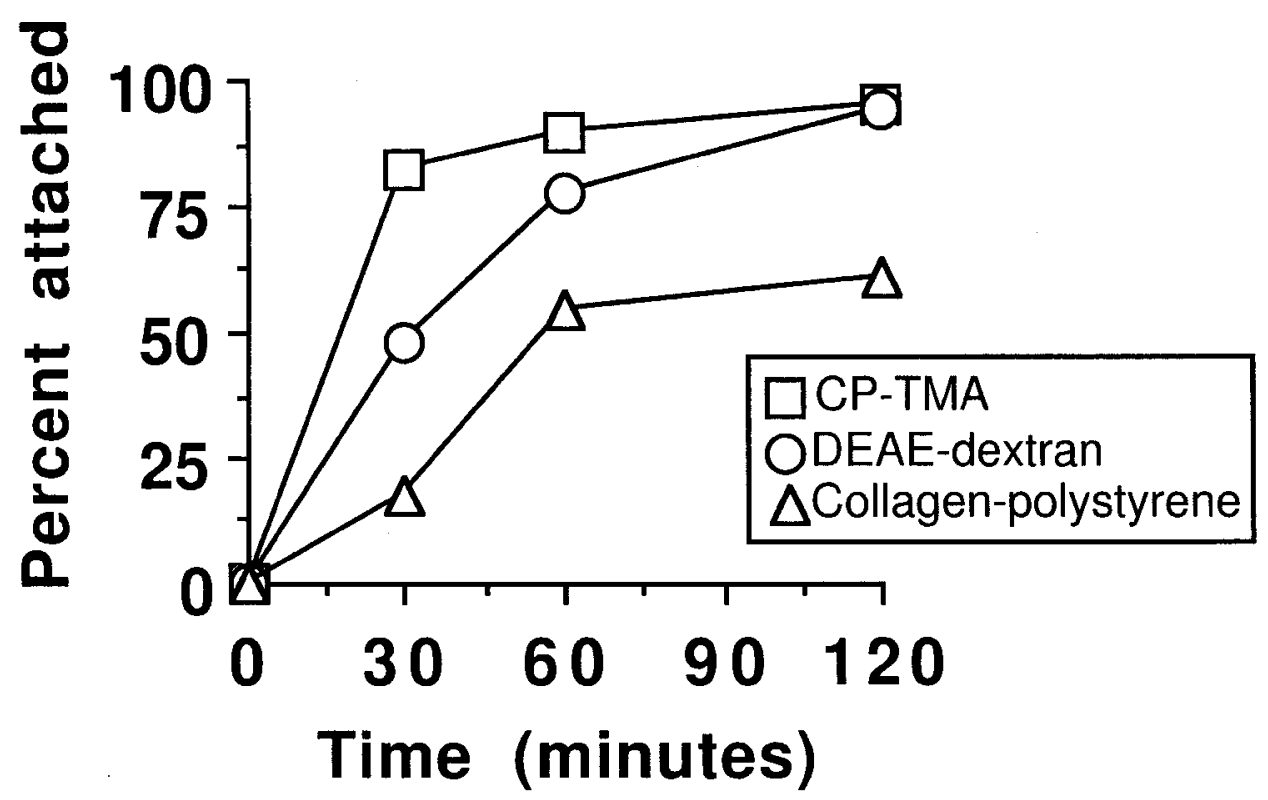

Figure 1. Attachment of MDBK cells to CP-TMA microcarriers: Comparison with DEAE-dextran and collagen-coated polystyrene microcarriers. The percent attached was determined by direct cell counts made following removal of the cells from the substrate with trypsin/EDTA. The data represent average cell counts where standard deviations were within $10 \%$ of mean values. The experiment was conducted three times with similar results.

rapidly attached to this substrate under serum-free conditions, and all of the cells rapidly spread after attachment. In previous studies it had been shown that while cells attached more rapidly to charged surface microcarriers than to collagen-coated microcarriers, cells spread more rapidly on collagen-coated polystyrene microcarriers after attachment than they did on the charged surface (Varani et al., 1995). Since the cells rapidly spread after attaching to the CPTMA microcarriers, this suggests that the CP-TMA microcarriers exhibit optimal adhesive characteristics of both the DEAE-dextran and collagen-coated polystyrene microcarriers.

\section{Cell growth on CP-TMA microcarriers}

In the next experiments, the four cell lines and the human diploid fibroblasts were examined for growth on the CP-TMA microcarriers under serum-free conditions. All of the cell lines grew to greater than $1 \times 10^{6}$ cells per $\mathrm{mL}$ within six days (Figure 2 ). Viable cell counts indicated greater than $90 \%$ viability in all cases (not shown). Phase-contrast and scanning electron microscopy showed that the microcarriers became completely covered with cells as the cell counts increased. Figure 3 shows a scanning electron micro- graph of CP-TMA microcarriers completely covered with Vero cells.

In contrast to results with the cell lines, low passage human diploid fibroblasts did not proliferate well on the CP-TMA microcarriers under serum-free conditions (Figure 2, middle-right). However, human diploid fibroblasts do not grow well in the absence of serum under any conditions and the same lack of growth was seen when these cells were grown in plastic cell culture dishes under the same conditions (not shown). To confirm that human diploid fibroblasts would grow on the CP-TMA microcarriers, the following experiment was peformed. Diploid fibroblasts were allowed to attach to CP-TMA microcarriers under serum-free conditions. One day later, the serumfree Aim V medium in half the culture dishes was replaced with DMEM-FBS. The other half received fresh Aim V medium. Cell counts made over the subsequent three day period indicated that proliferation did occur in the cultures to which serum-containing medium was added (Figure 2, lower-right). Thus, the failure of the human diploid fibroblasts to grow under serum-free conditions on the CP-TMA microcarriers was attributed to their serum-requirement rather than to incompatability with the substrate.

In a final set of experiments, small CP-TMA microcarriers (average diameter; 38-75 $\mu \mathrm{m}$ ) and 

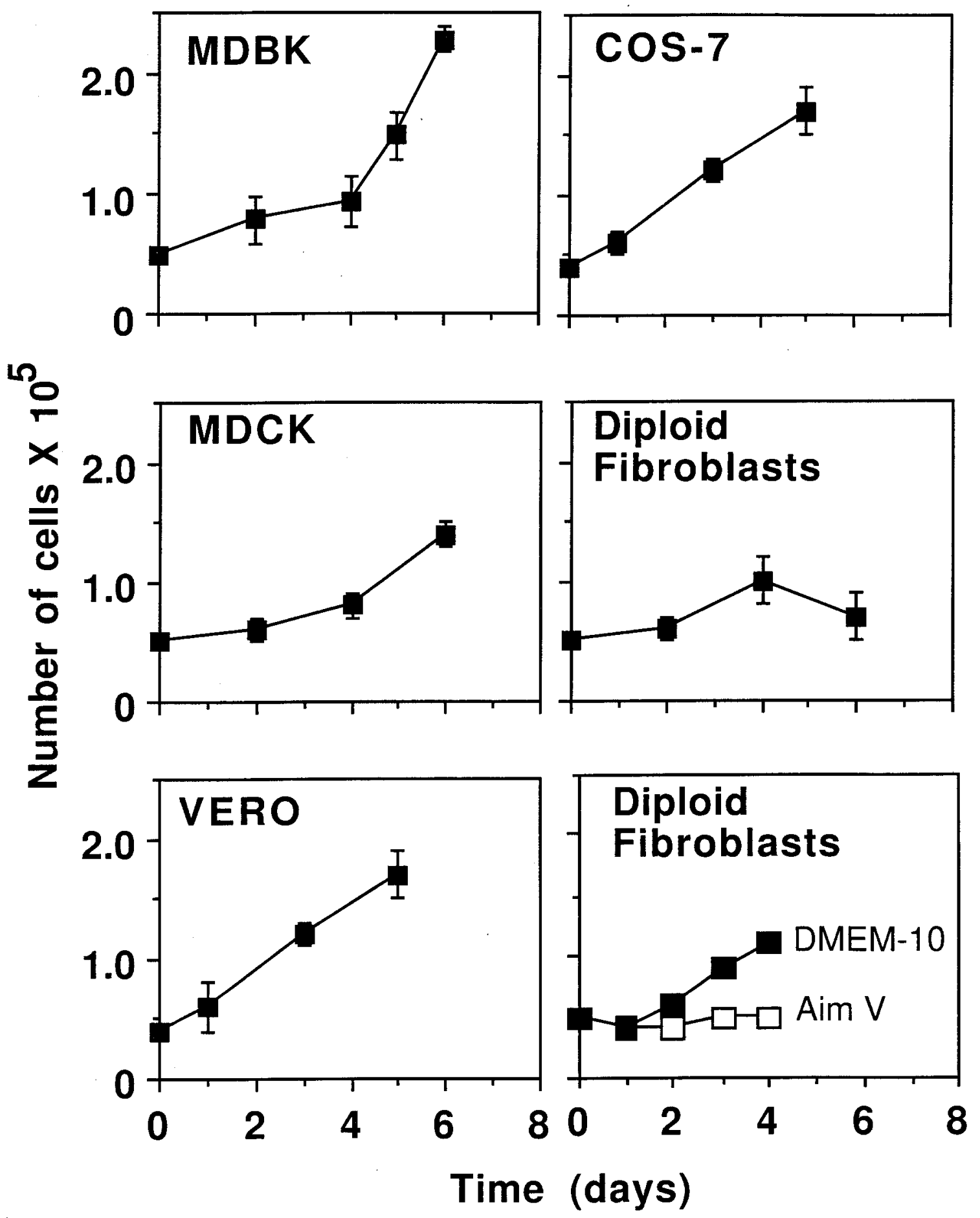

Figure 2. Cell Growth on CP-TMA microcarriers. Cells were added to $100-\mathrm{mL}$ suspension cultures at $4-5 \times 10^{5}$ cells per mL and grown for 6 days in serum-free Aim V medium (or DMEM-FBS as indicated). Fresh culture medium was provided every other day. The data represent average cell counts \pm s.d. based on triplicate or quadruplicate samples. Each cell type was examined at least three times with consistent results. 


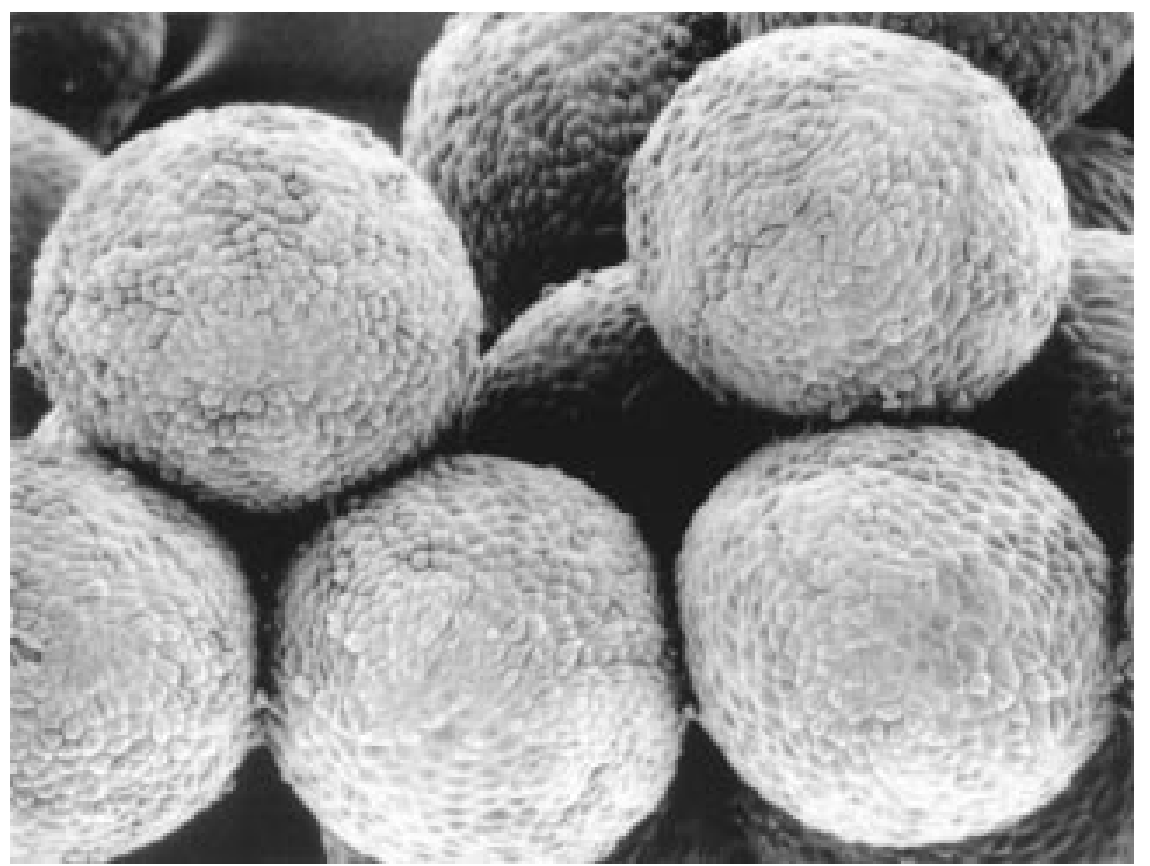

Figure 3. Scanning electron micrograph showing appearance of Vero cells at high-density on CP-TMA microcarriers (Magnification X600).

large CP-TMA microcarriers (average diameter; 150 $300 \mu \mathrm{m}$ ) were compared to the standard, intermediatesized CP-TMA microcarriers (average diameter; 75$150 \mu \mathrm{m}$ ) for ability to support attachment and growth of MDBK and Vero cells. As shown in Figure 4, when normalized for surface area, all three sizes of microcarriers performed equally well. This is of interest because previously it had been thought that microcarriers in the range of approximately 90-225 $\mu \mathrm{m}$ would be optimal with a fall-off in efficiency on either side of this range (Nielson and Johansson, 1980; Gebb, Clark and Hirtenstein, 1982; Obrenovitch, Maintier and Sene, 1982).

\section{Cell detachment from CP-TMA microcarriers}

In many large-scale cell culture applications, such as in the animal health industry, cells are never removed from the substrate. Rather, expressed products (such as the feline leukemia virus) are harvested from the culture fluid while cells, cell debris and substrate are discarded together. For such applications, ease of cell detachment from the substate is not an important consideration. However, production of other types of vaccines (for example, Marek's disease vaccine) requires that viable cells be released from the substrate in a viable state. Likewise, 'scale-up' protocols require removal of viable cells from the substrate. With this in mind, experiments were conducted in which MDBK cells, MDCK cells and human diploid fibroblasts were grown for one day on CP-TMA microcarriers. At the end of the incubation period, the culture medium (Aim V) was removed and the cells were exposed to trypsin/EDTA. Cells grown on DEAE-dextran and collagen-coated polystyrene microcarriers served as controls. Consistent with past findings (Varani et al., 1986), cells were readily released from the collagencoated polystyrene microcarriers but were much more resistant on the DEAE-dextran microcarriers (Figure 5). As can be seen from this figure, cell removal from CP-TMA microcarriers occurred as readily as from the CP-TMA microcarriers as from collagencoated polystyrene microcarriers.

\section{Production of infectious bovine rhinotracheitis virus by MDBK cells grown on CP-TMA microcarriers}

In the final series of experiments, MDBK cells were grown for one day on the CP-TMA microcarriers in serum-free Aim V medium. Cells grown on this substrate as well as on DEAE-dextran and collagenpolystyrene microcarriers were then infected with IBR virus. On the subsequent three days, duplicate cultures were harvested and assayed for infectious IBR virus. As shown in Figure 6, equivalent virus production occurred in cells grown on all three substrates. 


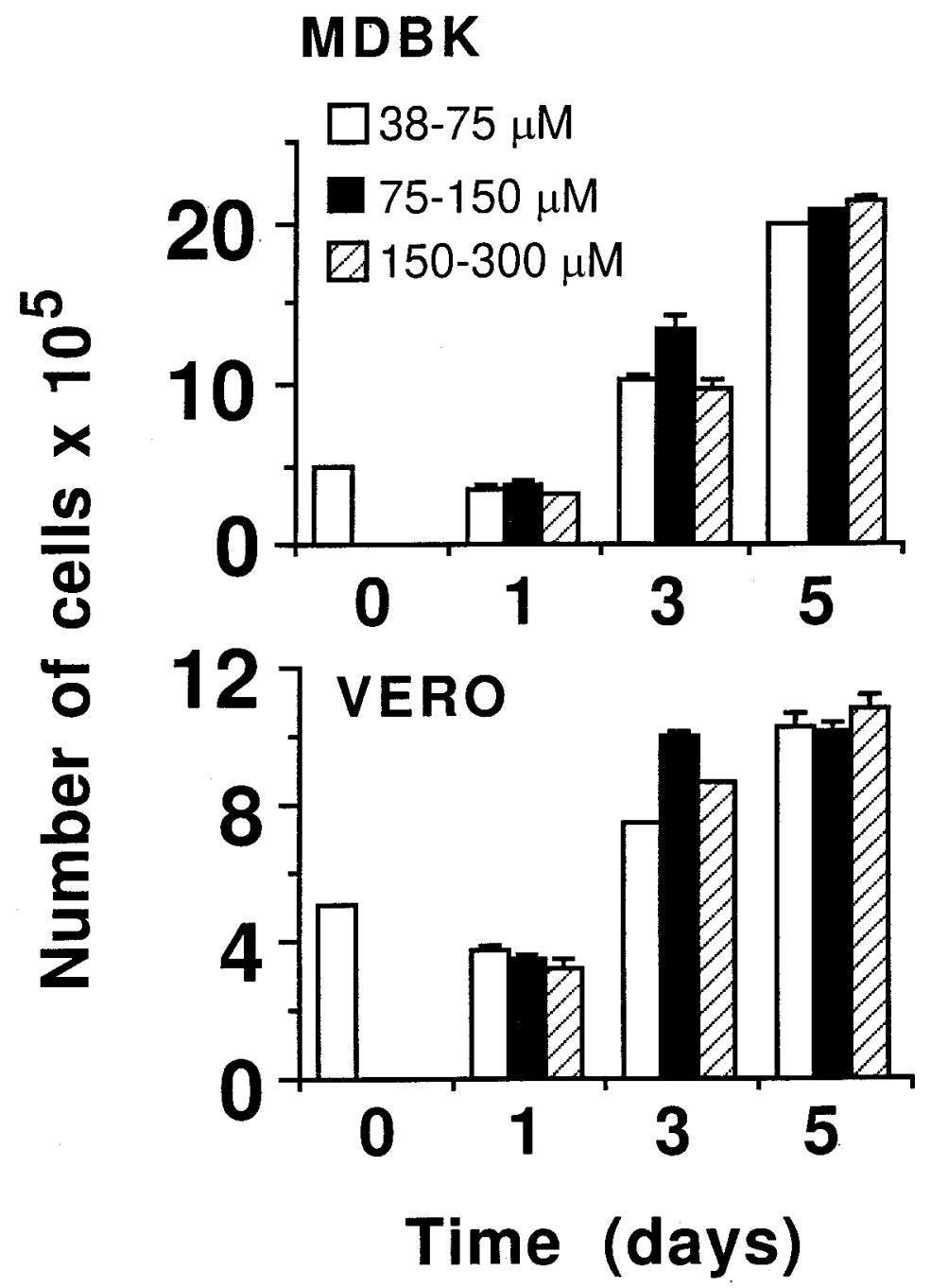

Figure 4. Growth of MDBK and Vero cells on CP-TMA microcarriers of three different sizes ranges. Cells were added to microcarriers at $5 \times 10^{5}$ cells per $\mathrm{mL}$ and grown for 5 days in serum-free Aim V. Fresh culture medium was provided every other day. The data represent average cell counts \pm s.d. based on triplicate samples. The experiment was repeated three times with similar results.

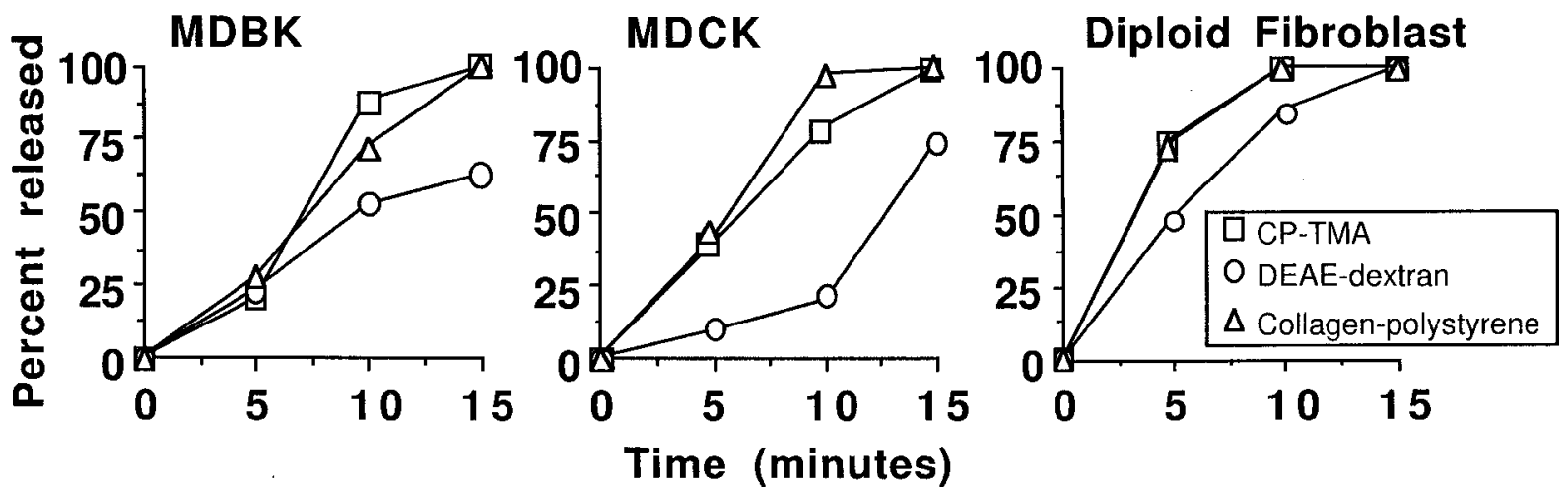

Figure 5. Release of cells from CP-TMA microcarriers in response to trypsin/EDTA. Comparison with release of cells from DEAE-dextran and collagen-coated polystyrene microcarriers. Cells grown for two days on each of the three substrates in Aim V medium were exposed to a solution of trypsin and EDTA. Released cells were separated from microcarriers and counted at each time point. The experiment was repeated three times with similar results. 


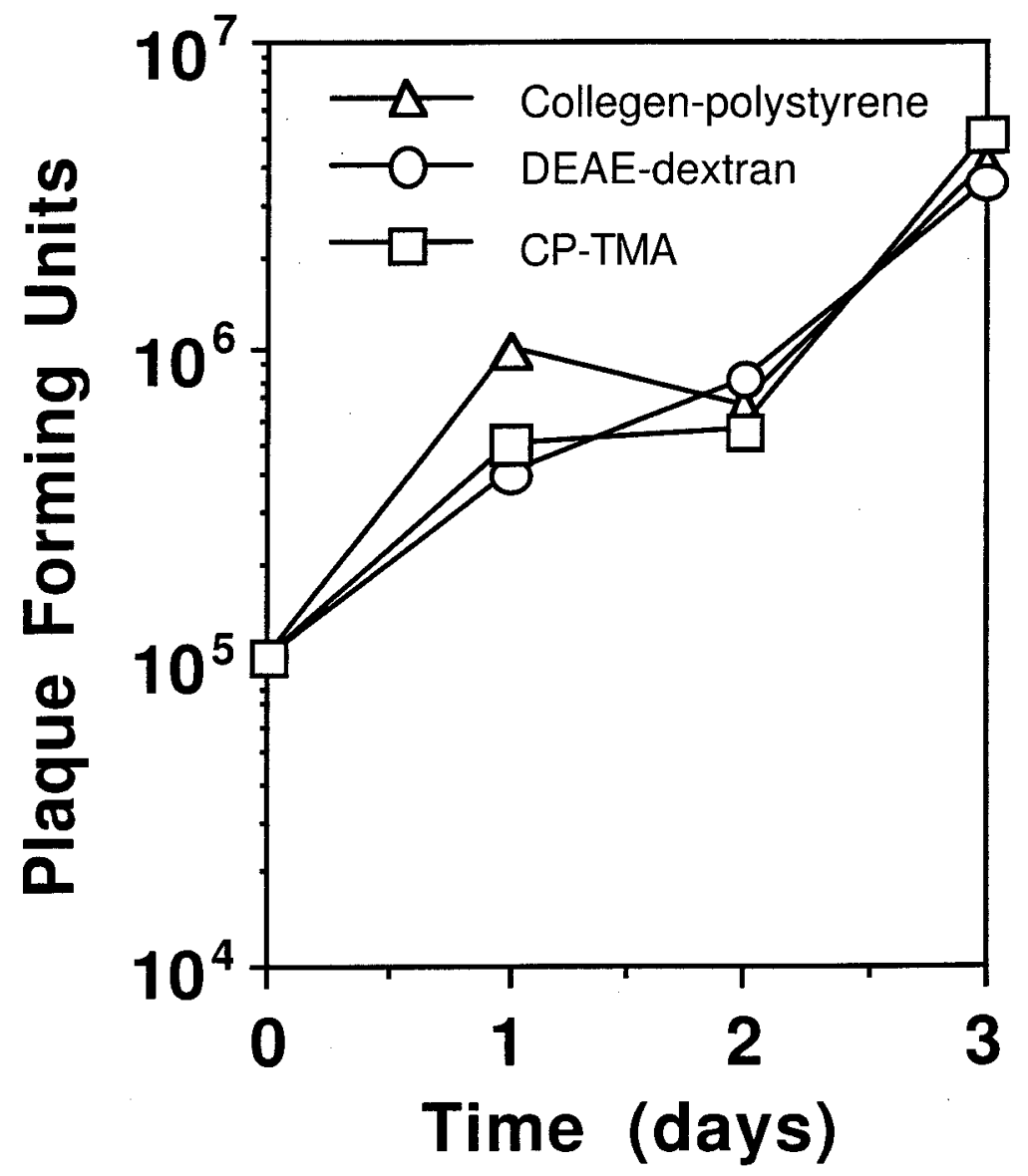

Figure 6. Production of infectious bovine rhinotracheitis (IBR) virus by MDBK cells grown on CP-TMA microcarriers. MDBK cells were grown for one day on CP-TMA microcarriers in serum-free Aim V medium. Collagen-coated polystyrene microcarriers and DEAE-dextran microcarriers served as controls. One day later, $1 \times 10^{5}$ plaque forming units (PFU) of IBR virus was added per $\mathrm{mL}$ to the flasks (approximately 0.5 multiplicity of infection). Samples were then obtained over the subsequent three day period and assayed for infectious virus. Values shown represent average number of plaque forming units, based on triplicate samples, where standard deviations were within $15 \%$ of the mean values. The experiment was repeated 3 times with similar results.

\section{Discussion}

At present, large-scale cultivation of anchoragedependent cells is done primarily for the production of vaccine strain viruses used in human and animal medicine. However, additional applications, including production of genetically-engineered biologicals, production of virus vectors for gene therapy and production of specific cell types for 'cellular therapies' - may utilize similar technology in the future. In the past, roller bottles have been the most extensively-used technology for large-scale cell production. More recently, other technologies including hollow fiber culture systems and microcarrier-bioreactor systems have replaced roller bottles in some applications. Hollow fiber reactors are useful for growing anchorage-independent cells or loosly adherent cells, but are not optimal for strongly-adherent cells. Microcarrier/bioreactor systems offer the best alternative to roller bottles for the large-scale cultivation of anchorage-dependent cells.

Microcarrier development got its start when Van Wezel (1967) demonstrated that DEAE-dextran beads could be used as a substrate for the growth of anchorage-dependent cells in a suspension culture mode. Since that time, a number of different materials including glass, polystyrene plastic, acrylamide, solid collagen, cellulose and liquid fluorocarbons have been successfully used as microcarriers (Giard, Thilly and Wang, 1977; Nielson and Johansson, 1980; Gebb, Clark and Hirtenstein, 1982; Obrenovitch, Maintier and Sene, 1982; Varani et al., 1983; Keese and Giaever, 1983). In addition, microcarriers have been 
produced which contain adhesion-supporting extracellular matrix components (or synthetic adhesionpromoting sequences) attached to the surface through covalent or non-covalent linkages (Varani et al., 1993, 1995). Although a large number of different material have been successfully used as microcarriers, only two types of products are widely-used in the industry today. These are charged-surface microcarriers (for example, DEAE-dextran) and matrix-coated microcarriers (for example, collagen - polystyrene or collagen - dextran).

Cells attach to charged-surface microcarriers primarily through electrostatic interactions, while interactions with extracellular matrix components on the microcarrier surface is mediated through specific cell surface receptors (Ginsburg, 1987; Swartz, 1993). Attachment to charged-surface microcarriers occurs, in general, more rapidly than to matrix-coated microcarriers. This is especially true with many of the transformed cell types used for vaccine manufacturing in the animal health industry, since these cells are often deficient in cell surface adhesion receptors (Plantefaber and Hynes, 1989). The high shear forces generated in suspension cultures further exaggerate the differences in attachment characteristics of the two types of microcarriers. Although cells initially attach to charged surfaces, cell spreading and cell growth can be retarded if the electrostatic charge is too great. Furthermore, the electrostatic interactions can prevent release of viable cells from the surface. In contrast, while initial attachment to the substrate through specific receptor-ligand interactions is less efficient, cells rapidly spread and begin proliferating after attachment (Varani et al., 1995).

The CP-TMA microcarriers described here are unique in that they express desirable characteristics of both the charged-surface and matrix-coated microcarriers. A variety of cell types rapidly attach to this material in suspension culture (under serum-free conditions). Once attached, the cells rapidly spread and rapidly begin proliferating. Cells grow to high density, but at the end of the growth phase they can be easily released from the substrate by exposure to trypsin/EDTA. Given these characteristics of the CPTMA microcarriers, this substrate may provide an ideal replacement for currently available substrates for use in large-scale, serum-free culture systems.

\section{Acknowledgement}

This study was supported in part by grant CA 74595 from the USPHS. The authors would like to thank Ms. Sue Ann Stamper for assistance with scanning electron microscopy.

\section{References}

Gebb C, Clark JM and Hirtenstein MD (1982) Alternate surface for microcarrier culture of animal cells. Dev Biol Standards 50: 93102.

Giard DJ, Thilly WG and Wang DIC (1977) Virus production with a newly-developed microcarrier system. Appl Environ Microbiol 34: 668-672.

Ginsburg I (1987) Cationic polyelectrolytes: A new look at their possible role as opsinins, as stimulators of the respiratory burst in leukocytes, in bacteriolysis and as modulators of immune complex disease. Inflammation 11: 489-495.

Keese O and Giaever I (1983) Cell growth on liquid microcarriers. Science 219: 1448-1449.

Nielson V and Johnson A (1980) Biosilon: Optimal culture conditions and various research scale culture techniques. Dev Biol Stand 46: 131-136.

Obrenovitch A, Maintier C and Sene C (1982) Microcarrier culture of fibroblastic cells on modified trisacryl beads. Biol Cell 46: 249-256.

Plantefaber LC and Hynes RO (1989) Changes in integrin receptors on oncogenically transformed cells. Cell 56: 281-290.

Ruoslahti E and Pierschbacher MD (1986) Arg-Gly-Asp: A versitile cell recognition signal. Cell 44517-518.

Swartz MA (1993) Signaling by integrins; implications for tumorigenesis. Cancer Res 53: 1503-1506.

Tamm I, Kikuchi T, Wang E and Pfeffer LM (1984) Growth rate of control and b-interferon-treated human fibroblast populations over the course of their in vitro life-spans. Cancer Res 44: 22912296.

Van Wezel AL (1967) Growth of cell strains and primary cells on microcarriers. Nature 216: 65-66.

Varani J, Dame M, Beals TF and Wass JA (1983) Growth of three established cell lines on glass microcarriers. Biotech Bioengineer 25: 1359-1372.

Varani J, Bendelow MJ, Chun JH and Hillegas WJ (1986) Cell growth on microcarriers: Comparison of proliferation on and recovery from various substrates. J Biol Stand 14: 331-336.

Varani J, Fligiel SEG, Inman DR, Helmreich DL, Bendelow MJ and Hillegas WJ (1989) Substrate-dependent differences in production of extracellular matrix molecules by squamous carcinoma cells and diploid fibroblasts. Biotech Bioengineer 33: 12351241.

Varani J, Inman DR, Fligiel EGS and Hillegas WJ (1993) Use of recombinant and synthetic peptides as attachment factors for cells on microcarriers. Cytotechnology 13: 89-98.

Varani J, Fligiel SEG, Inman DR, Beals TF and Hillegas WJ (1995) Modulation of adhesive properties of DEAE dextran with laminin. J Biomaterials Res 23: 993-997. 